\title{
PROPERTIES OF PROPAGATION OF QUANTUM SPIN SYSTEMS
}

\author{
DEREK W. ROBINSON
}

(Received 31 May 1976)

\begin{abstract}
Using an estimate on the group velocity we give an independent proof of the existence of time translations for a large class of short range interactions. We demonstrate that these systems satisfy a strong form of causal propagation and that space-time algebras in suitable space-like directions are disjoint. Finally we derive criteria for dispersion of the interaction in terms of the algebraic density of the orbit of local subalgebras under the evolution or under the associated group of shifts. In this sense the Heisenberg and $X-Y$ models are dispersive but the Ising model is not.
\end{abstract}

\section{Introduction}

In this paper we analyze various properties of time translations of quantum spin systems. This analysis falls into two parts. First we consider various structural properties which follow from the fact that the system has a finite group velocity, [1], i.e. high velocity components are virtually absent. Secondly we investigate criteria for separating dispersive interactions from non-dispersive interactions. In this respect it is apparently useful to consider space-time algebras built from the orbits of local subalgebras. These algebras are analogues of the partitions generated in classical ergodic theory by translation of a fixed finite partition [2]. If these orbit subalgebras are 'large' the system is in some sense dispersive.

\section{Group velocity estimates}

We use the notation and definitions of [1] and [3]. In particular an interaction $\Phi$ is a function from the finite sets $X \subset Z^{\prime}$ to hermitian elements $\Phi(X) \subset \mathfrak{A}_{X}$. We consider those functions which satisfy the condition 


$$
\|\Phi\|_{\gamma}=\sup _{x \in \mathbf{z}^{\nu}} \sum_{X \ni x} N(X) N^{2 N(X)} e^{\gamma D(X)}\|\Phi(X)\|<+\infty
$$

where $N(X)$ is the number of points in the set $X, D(X)$ is the diameter of this set, $N$ is the dimension of the vector space associated with each point $x \in Z^{\nu}$, and $\gamma>0$. These interactions form a Banach space $B_{\gamma}$ with respect to the norm $\|-\|_{\gamma}$.

For each $\Lambda \subset \mathbb{Z}^{\nu}$ and $A \in \mathfrak{A}$ we introduce the notation

$$
\tau_{i}^{\Phi, \Lambda}(A)=e^{u H_{\Phi}(\Lambda)} A e^{-ı H_{\Phi}(\Lambda)}, \Phi \in B_{\gamma}, t \in \mathbb{R}
$$

where

$$
H_{\Phi}(\Lambda)=\sum_{X \subset} \Phi(X)
$$

The following estimation is a generalization of that derived in [1] but we present its proof in a slightly different form.

Proposition 1. If $A \in \mathfrak{U}_{\{0\}}$ and $B \in \mathfrak{U}$ then

$$
\left\|\left[\tau_{1}^{\Phi, \wedge}(A), B\right]\right\| \leqq\|A\| \sum_{x \in \mathbf{Z}^{*}} \sup _{A \in \mathbb{R}_{0 \mid}} \frac{\left\|\left[\tau_{x}(A), B\right]\right\|}{\|A\|} e^{-|x| \gamma+2|+|\|\Phi\|_{\gamma}}
$$

for each $\Phi \in B_{r}$.

Proof. First define

$$
C_{A, B}^{\wedge}(x, t)=\left[\tau_{x} \tau_{t}^{\Phi, \wedge}(A), B\right]
$$

then clearly one has

$$
\begin{aligned}
C_{A, B}^{\wedge}(x, t) & =C_{A, B}^{\wedge}(x, 0)+\int_{0}^{1} d s \frac{d}{d s} C_{A, B}^{\wedge}(x, s) \\
& =C_{A, B}^{\wedge}(x, 0)+i \sum_{x \ni 0} \int_{0}^{1} d s\left[\tau_{x} \tau_{s}^{\Phi, \wedge}([\Phi(X), A]), B\right] .
\end{aligned}
$$

Next define

$$
F(x, t)=\sup _{A \subset Z^{\prime}} \sup _{A \in Q_{\{0\}}}\left\|C_{A . B}^{\wedge}(x, t)\right\| /\|A\| .
$$

From the integral relation for $C_{A, B}^{\wedge}$ one then deduces the following integral inequality for $F$.

$$
F(x, t) \leqq F(x, 0)+\sum_{X \ni 0} \int_{0}^{\prime \prime 4} d s \sup _{\Lambda, A}\left\|\left[\tau_{x} \tau_{s}^{\Phi, \wedge}([\Phi(X), A]), B\right]\right\| /\|A\| .
$$

Now we use the methods of [1] to majorize the integrand in terms of $F$. Let $e\left(i_{x}, j_{x}\right), i_{x}, j_{x}=1,2, \cdots N$ be a set of matrix units for $\mathscr{K}_{\{x\}}$, i.e. 


$$
e\left(i_{x}, j_{x}\right) e\left(k_{x}, l_{x}\right)=\delta_{l x} k_{x} e\left(i_{x}, l_{x}\right) \text {, etc. }
$$

In terms of such units $\Phi(X)$ has a unique decomposition

$$
\Phi(X)=\sum_{\left.\left.\left\{t_{x}\right\}\right\}_{x}\right\}} C_{\Phi}\left(\left\{i_{x}\right\},\left\{j_{x}\right\}\right) \prod_{x \in X} e\left(i_{x}, j_{x}\right)
$$

with coefficients $C_{\Phi} \in \mathbb{C}$ satisfying

$$
\left|C_{\Phi}\left(\left\{i_{x}\right\},\left\{j_{x}\right\}\right)\right| \leqq\|\Phi(X)\| .
$$

Substitution of this decomposition into the integrand of the above differential inequality, use of the linearity properties of commutators, and the triangle inequality give the new integral inequality

$$
F(x, t) \leqq F(x, 0)+\sum_{X \ni 0} \sum_{y \in X} 2 N^{2 N(x)}\|\Phi(X)\| \int_{0}^{|t|} d s F(x+y, s) .
$$

Introducing $\omega$ by the definition

$$
\omega(y)=\sum_{\substack{x \ni 0 \\ x \ni y}} N^{2 N(x)}\|\Phi(X)\| .
$$

This integral inequality takes the final simple form

$$
F(x, t) \leqq F(x, 0)+2 \sum_{y \in \mathbf{z}^{\nu}} \omega(y) \int_{0}^{|x|} d s F(x+y, s) .
$$

Iteration of this inequality then gives

$$
F(x, t) \leqq \sum_{y \in \mathbf{Y}^{\nu}} F(y, 0) \frac{1}{(2 \pi)^{\nu}} \int_{0}^{2 \pi} d \theta_{1} \cdots \int_{0}^{2 \pi} d \theta_{\nu} e^{i(x-y) \theta} e^{2|t| \tilde{\omega}(\theta)}
$$

where $\tilde{\omega}$ is the Fourier transform of $\omega$.

Finally one can proceed as in [1] and shift the integration to an integration along $\operatorname{Im} \theta=\hat{\gamma}$ and then one finds the bound

$$
F(x, t) \leqq \sum_{y \in Z^{\nu}} F(y, 0) e^{-(x-y) \hat{\gamma}} e^{2|t| \hat{\omega}(\dot{y})} .
$$

But choosing $\hat{\gamma}=\gamma \frac{x-y}{|x-y|}$ one has

$$
\begin{aligned}
\tilde{\omega}(i \hat{\gamma}) & =\sum_{X \ni 0} \sum_{y \in X} N^{2 N(X)}\|\Phi(X)\| e^{\gamma(x-y) y /|x-y|} \\
& \leqq \sum_{X \ni 0} N(X) N^{2 N(X)}\|\Phi(X)\| e^{\gamma D(X)} \\
& =\|\Phi\|_{\gamma} .
\end{aligned}
$$


Thus

$$
F(x, t) \leqq \sum_{y \in \mathbf{x}^{\nu}} F(y, 0) e^{-\gamma|x-y|} e^{2|1|\|\oplus\|_{\gamma}}
$$

and the result of the proposition follows by use of the definition of $F$.

Corollary 1. If $A, B \in \mathfrak{A}_{\{0\}}, \Phi \in B_{\gamma}$ then

$$
\left\|\left[\tau_{x} \tau_{1}^{\Phi, A}(A), B\right]\right\| \leqq 2\|A\|\|B\| e^{-|x| y+2|\cdot||| \Phi \|_{\gamma}} .
$$

The proof follows by noting that $\left\|\left[\tau_{x}(A), B\right]\right\| \leqq 2\|A\|\|B\| \delta_{x, 0}$.

Corollary 2. If $A \in \mathfrak{A}_{\Lambda_{0}}$ and $B \in \mathfrak{U}$ then

$$
\left\|\left[\tau_{1}^{\Phi, \Lambda}(A), B\right]\right\| \leqq\|A\| \sum_{x \in \mathbf{Z}^{\prime}} \sup _{A \in 2_{\{0\}}} \frac{\left.\| \tau_{x}(A), B\right] \|}{\|A\|} N^{N\left(\Lambda_{0}\right)} \sum_{y \in \Lambda_{0}} e^{-|x-y| y+2|t|\|\Phi\|_{\gamma}}
$$

for each $\Phi \in B_{\gamma}$.

This corollary follows by expressing $A$ in terms of matrix units of the type used in the proof of the proposition and then using linearity properties of the commutator and the triangle inequality.

\section{Causal propagation}

The commutator estimates given in the previous section can be used to give information about the automorphisms $\tau^{\Phi, \Lambda}$ and their limits as $\Lambda \rightarrow \infty$.

THEOREM 1. If $A \in \mathfrak{A}_{\Lambda_{0}}$, where $0 \in \Lambda_{0}$, and $\Phi \in B_{y}$ then

$$
\left\|\tau_{1}^{\Phi, \Lambda_{2}}(A)-\tau_{1}^{\Phi, \Lambda_{1}}(A)\right\| \leqq\|A\| N\left(\Lambda_{0}\right)\left[\frac{e^{2|t|\|\Phi\|_{\gamma}}-1}{\|\Phi\|_{\gamma}}\right]\left[\sum_{x \in \Lambda_{2} \mid \Lambda_{1}} e^{-|x|_{0} \gamma}\right] .
$$

Further, if $\Phi_{1}, \Phi_{2} \in B_{\gamma}$ then

$$
\left\|\tau_{1}^{\Phi_{1} \cdot A}(A)-\tau_{1}^{\Phi_{2} \cdot A}(A)\right\| \leqq\|A\| N\left(\Lambda_{0}\right)\left\|\Phi_{1}-\Phi_{2}\right\|_{\gamma}\left[\frac{e^{2|\iota|\|\Phi\|_{\gamma}}-1}{\|\Phi\|_{\gamma}}\right]\left[\sum_{x \in \mathbf{z}^{*}} e^{-|x|_{0} \gamma}\right]
$$

where $\|\Phi\|_{\gamma}=\min _{\imath=1,2}\left(\left\|\Phi_{1}\right\|_{\gamma},\left\|\Phi_{2}\right\|_{\gamma}\right)$.

Proof. To prove the first statement we use the integral relation

$$
\tau_{-i}^{\Phi, \Lambda_{2}} \tau_{1}^{\Phi, \Lambda_{1}}(A)-A=-i \int_{0}^{1} d s \tau_{-s}^{\Phi, \Lambda_{2}}\left(\left[H_{\Phi}\left(\Lambda_{2}\right)-H_{\Phi}\left(\Lambda_{2}\right), \tau_{s}^{\Phi, \Lambda_{1}}(A)\right]\right)
$$

Thus

$$
\left\|\tau_{1}^{\Phi_{1} A_{2}}(A)-\tau_{1}^{\Phi, A_{1}}(A)\right\| \leqq \sum_{x \in \Lambda_{2} \mid \Lambda_{1}} \sum_{X \ni x} \int_{0}^{1 t^{\prime}} d s \|\left[\Phi(X), \tau_{s}^{\Phi, \Lambda_{1}}(A) \|\right.
$$


Using the decomposition of $\Phi(X)$ introduced in Section 2 and exploiting the linearity of the commutators in the same manner one finds

$$
\begin{aligned}
& \left\|\tau_{t}^{\Phi, \Lambda_{2}}(A)-\tau_{t}^{\Phi, \Lambda_{1}}(A)\right\| \leqq\|A\| \sum_{x \in \Lambda_{2} \mid \Lambda_{1}} \sum_{X \ni x} N^{2 N(x)}\|\Phi(X)\| \sum_{y \in X} \int_{0}^{\mid \prime \prime} d s F(y,-s) \\
& \leqq\|A\| \sum_{x \in \Lambda_{2} \backslash \Lambda_{1}} \sum_{X \ni_{x}} N^{2 N(x)}\|\Phi(X)\| \cdots \\
& \cdots 2 \sum_{y \in X} \sum_{z \in \Lambda_{0}} e^{-\gamma|y-z|} \int_{0}^{|0|} d s e^{2 s\|\Phi\|_{\gamma} !}
\end{aligned}
$$

But $-|y-z| \leqq-|x-z|+|x-y|$ and $|x-y| \leqq D(X)$ for $x, y \in X$. The first result follows immediately.

A similar argument yields the inequality

$$
\left\|\tau_{t}^{\Phi_{1}, \wedge}(A)-\tau_{1}^{\Phi_{2} \wedge}(A)\right\| \leqq \int_{0}^{|x|} d s \sum_{x \in \mathbf{Z}^{\nu}} \sum_{X \ni x} \|\left[\Phi_{1}(X)-\Phi_{2}(X), \tau_{s}^{\left.\Phi_{i, \wedge}(A)\right] \|}\right.
$$

for $i=1$ or 2 . The second statement follows by application of the same estimation procedure.

The first statement of the above theorem establishes the existence of the group of automorphisms of $\mathfrak{H}$, which corresponds to time translations, and is defined by the limits

$$
\tau_{\imath}^{\Phi}(A)=\lim _{\Lambda \rightarrow \infty} \tau_{1}^{\Phi, A}(A) \quad A \in \mathfrak{N}, t \in \mathbb{R} .
$$

The second statement establishes the continuity of this group on $\mathbb{R} \times B_{\gamma}$. The above limits exist in the uniform topology of the $C^{*}$ algebra 9 whenever $\Lambda \rightarrow \infty$ in the sense that $\Lambda$ eventually contains each compact $\Lambda^{\prime} \subset \mathbb{Z}^{\nu}$. This proof is independent of that previously known [3] [4] and gives much better approximations for $\tau_{1}^{\Phi}$ when $t$ is real. It fails, however, to give information about the analyticity of $t \rightarrow \tau_{t}^{\Phi}$ for complex $t$. These estimates give the following statement about the causal propagation of the system.

THEOREM 2. Take $\Lambda_{0} \subset \mathbb{Z}^{\nu}, \Phi \in B_{\gamma}$, and define $V_{\Phi}=2\|\Phi\|_{\gamma} / \gamma$,

$$
\Lambda_{R}=\left\{x ; x \in Z^{\nu}, \min _{y \in \Lambda_{0}}|x-y|>R\right\} .
$$

There is a polynomial $P_{\nu-1}$, of order $\nu-1$, independent of $\Phi$ and $t$, such that

$$
\begin{aligned}
& \sup _{A \in \mathscr{T}_{A_{0}}}\left\|\tau_{i}^{\Phi}(A)-e^{\left.u H_{\Phi}^{(A} \Lambda_{V \mid l+\infty}\right)} A e^{-u H_{\Phi}\left(A_{V|l|+D}\right)}\right\| /\|A\| \\
& \leqq 2|t| e^{-|t|\left(v-v_{\phi}\right)} P_{\nu-1}(\gamma(V|t|+D)) e^{-\gamma D} / \gamma^{\nu} .
\end{aligned}
$$


In particular for $\varepsilon>0$, and $V>V_{\Phi}$, there is a $D$ such that

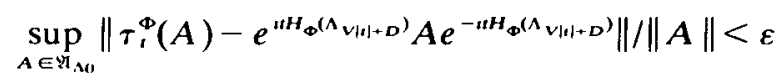

for all $t \in \mathbb{R}$.

Proof. The first statement follows from Theorem 1 by using the definition of $\tau_{t}^{\Phi}$, the fact that

$$
\left(e^{2|||| \mid \phi \Phi \|_{\gamma}}-1\right) /\|\Phi\|_{\gamma} \leqq 2|t| e^{2|t|\|\Phi\|_{\gamma}},
$$

and the estimate

$$
\sum_{x \in \mathbf{Z}^{\nu},|x|_{0}>R} e^{-\gamma|x|_{0}} \leqq P_{\nu-1}(\gamma R) e^{-\gamma R} / \gamma^{\nu}
$$

The last bound simply follows by majorising the sum with an integral over $\mathbb{R}^{\nu}$. The second statement is deduced by noting (1) for any $\alpha>0$ there is a $C_{\alpha}$ such that $P_{\nu-1}(x)<C_{\alpha} e^{\alpha x},(2)$ one can choose $1>\alpha>0$ such that $(1-\alpha) V>V_{\Phi}$, and (3) the function $x \in \mathbb{R} \rightarrow|x| e^{-|x|}$ is uniformly bounded.

The result of this theorem can be interpreted in two physically equivalent ways. One could interpret the $\tau_{i}^{\Phi}(A), A \in \mathfrak{P}_{\Lambda_{0}}$ and $t>0$, as the observables in the region $\Lambda_{0}$ at time $t$ and one then sees that these observables are determined, up to $\varepsilon$, by the observables in the region $\Lambda_{v t+D}$ at time zero, i.e. there is a causal propagation with a maximum velocity $V_{\Phi}^{\prime}=\min _{\gamma} 2\|\Phi\|_{\gamma} / \gamma$. Alternatively one could say that the effects of an operation $A$ in $\Lambda_{0}$ at time zero only propagate in the cone $|x| \leqq V t+D$. Thus $V_{\Phi}^{\prime}$ is an upper bound on the velocity of propagation, i.e. the group velocity.

\section{Space-time algebras}

The subalgebras $\mathfrak{A}_{\Lambda}$ of $\mathfrak{U}$ are interpretable as the algebra of observables in the region $\Lambda \subset \mathbb{Z}^{\nu}$ at time $t=0$. It is natural to interpret the algebras $\mathfrak{I}_{\Lambda . t}^{\phi}$ defined for $\Phi \in B_{\gamma}$ by

$$
\mathfrak{P}_{\Lambda, t}^{\Phi}=\left\{\tau_{\mathfrak{1}}^{\Phi}(A) ; A \in \mathfrak{N}_{\Lambda}\right\},
$$

as the algebra of observables in $\Lambda$ at time $t$. The foregoing estimates allow one to conclude that the algebras $\mathfrak{H}_{\Lambda_{1,1},}^{\Phi}$ and $\mathfrak{H}_{\Lambda_{2}, t_{2}}^{\Phi}$ commute approximately whenever the distance $d\left(\Lambda_{1}, \Lambda_{2}\right)$ between $\Lambda_{1}$ and $\Lambda_{2}$ is large compared to $\left|t_{1}-t_{2}\right|$. A different statement of this independence is given as follows.

THEOREM 3. Adopt the above notation and take $\Phi \in B_{\gamma}$. It follows that

$$
\mathfrak{Y}_{\Lambda_{1}, t_{1}}^{\infty} \cap \mathfrak{A}_{\Lambda_{2}, t_{2}}^{\infty}=\{\lambda \mathbb{1}\}
$$


where $\mathbb{1}$ is the identity of $\mathfrak{I}$ and $\lambda \in \mathbb{C}$, whenever

$$
d\left(\Lambda_{1}, \Lambda_{2}\right)>\left|t_{1}-t_{2}\right| \frac{2\|\Phi\|_{\gamma}}{\gamma}+\frac{1}{\gamma} C\left(\left|\Lambda_{1}\right| ;\left|\Lambda_{2}\right|\right)
$$

where $C\left(\Lambda_{1}, \Lambda_{2}\right) \in \mathbb{R} \cdot$ is independent of $\Phi$.

Proof. If $A_{1} \in \mathfrak{P}_{\Lambda_{1}, t_{1}}^{\Phi}$ and $A_{2} \in \mathfrak{M}_{\Lambda_{2}, t_{2}}^{\Phi}$ then the proposition of section 2 gives the estimate

$$
\left\|\left[A_{1}, A_{2}\right]\right\| \leqq 2\left\|A_{1}\right\|\left\|A_{2}\right\| N\left(\Lambda_{1}\right) N^{N\left(\Lambda_{2}\right)} e^{-d\left(\Lambda_{1}, \Lambda_{2}\right) \gamma+2\left|t_{1}-t_{2}\right|\|\Phi\|_{\gamma}}
$$

Now take $B=B^{+} \in \mathfrak{Y}_{\Lambda_{1, t 1}}^{\Phi} \cap \mathfrak{Y}_{\Lambda_{2}, t_{2}}^{\Phi}$ with $B \notin \lambda \mathbb{1}$. Thus $B \in \mathfrak{A}_{\Lambda_{1}, 1_{1}}^{\Phi}$. Next we will construct a $B^{\prime}$, with the same properties as $B$, and an $A \in \mathfrak{P}_{\Lambda_{1}, l_{1}}^{\infty}$ such that

$$
\left\|\left[A, B^{\prime}\right]\right\|=2\|A\|\left\|B^{\prime}\right\| .
$$

To do this let $U \in \mathfrak{Y}_{\Lambda_{1}, r_{1}}^{\Phi}$ be a unitary matrix which brings $B$ into diagonal form. Let $\lambda_{M}, \lambda_{m}$ be the largest and smallest eigenvalues of $B$. Then we have the following matrix representation

$$
B^{\prime \prime}=\frac{2}{\lambda_{M}-\lambda_{m}}\left(U B U^{-1}-\frac{\left(\lambda_{M}+\lambda_{m}\right) \mathbb{1}}{2}\right)=\left(\begin{array}{rrrrr}
1 & & & \\
& -1 & & 0 \\
& & \lambda_{1} & \\
& & & \lambda_{2} \\
& & & \\
& 0 & & & \\
& & & & \\
& & & &
\end{array}\right)
$$

with $\left|\lambda_{1}\right| \leqq 1$. Now choose $A$ to be given by

$$
A^{\prime}=\left(\begin{array}{lll}
0 & 1 & \\
1 & 0 & 0 \\
& 0 & 0
\end{array}\right)
$$

It is readily checked that $A^{\prime}$ and $B^{\prime \prime}$ have the desired properties.

Finally if

$$
B^{\prime}=U^{-1} B^{\prime \prime} U, A=U^{-1} A^{\prime} U
$$

then $B^{\prime}$ and $A$ have the desired properties. But we also have

$$
B^{\prime}=\frac{2}{\lambda_{M}-\lambda_{m}}\left[B-\frac{\left(\lambda_{M}+\lambda_{m}\right) \mathbb{H}}{2}\right] \in \mathfrak{H}_{\Lambda_{2, t_{2}}}^{\Phi} \text {. }
$$


Thus

$$
2\|A\|\left\|B^{\prime}\right\| \leqq 2\|A\|\left\|B^{\prime}\right\| N\left(\Lambda_{1}\right) N^{N\left(\Lambda_{2}\right)} e^{-d\left(\Lambda_{1}, \Lambda_{2}\right) r+2||_{2}-\varepsilon_{1}\|\not\|_{\gamma}} .
$$

This inequality gives a contradiction if

$$
d\left(\Lambda_{1} ; \Lambda_{2}\right)>2\left|t_{1}-t_{2}\right| \frac{\|\Phi\|_{\gamma}}{\gamma}+\frac{1}{\gamma} \log N\left(\Lambda_{1}\right) N^{N\left(\Lambda_{2}\right)}
$$

and the theorem is proved.

\section{Orbits, shifts and dispersion}

The properties of propagation discussed in the previous sections, follow from the effective suppression of high velocities. This phenomenon is a direct effect of the short range interaction and these results are valid for all interactions of the class $B_{\gamma}$ independently of their detailed structure. In this concluding section we wish to make several remarks about dispersive and non-dispersive propagation.

The space $B_{\gamma}$ contains interactions of a 'classical' nature which lead to an oscillatory time behaviour and also interactions of a more 'quantum mechanical' type which give rise to dispersive phenomena. The principal problem is to isolate, and characterize, the latter interactions. One property which has been suggested as a characterization of a dispersive interaction is the property of asymptotic abelianness, i.e.

$$
\lim _{\mid a t \rightarrow \infty}\left\|\left[A, \tau_{:}^{\Phi}(B)\right]\right\|=0, A, B \in \Re .
$$

Although this condition is both natural and useful it is apparently very difficult to verify for any given $\Phi$. Thus it is logical to search for other characterizations.

Various possibilities can be formulated in terms of the space-time algebras of Section 4. If, for example, $\tau^{\Phi}$ leaves certain elements $A \in \mathfrak{I}_{\text {A }}$ invariant (with $A \notin \lambda \mathbb{1}$ ) then $\mathfrak{I}_{\Lambda_{, t_{1}}} \cap \mathfrak{P}_{\Lambda_{, t_{2}}}$ is non-trivial for all $t_{1}, t_{2} \in \mathbb{R}$. If, alternatively, $\tau_{1}^{\Phi}(A)$ is oscillatory in nature then $\mathfrak{I}_{A . t_{1}} \cap \mathfrak{P}_{\Lambda, t_{2}}$ will be nontrivial for certain $t_{1}, t_{2} \in \mathbb{R}$. These properties are verified for classical interactions of the Ising type and are characteristic of non-dispersion. In these cases algebras of the type $\cap, \mathfrak{H}_{\Lambda, t_{1}}$ tend to be small, consisting only of elements localized in $\Lambda$. The behaviour is quite different for non-dispersive interactions. To formulate this alternative behaviour it is useful to define various generalizations of the space-time algebras of Section 4.

Let $G=\{A, B, C, \cdots\}$ be a subset of a $C^{*}$-algebra $: \mathbb{t}$ and let $\tau$ denote a strongly continuous one-parameter group of ${ }^{*}$-automorphisms of $\mathfrak{A}$. We 
introduce a $C^{*}$-subalgebra $\mathfrak{A}_{I}(G)$ of $\mathfrak{A}$ parametrized by the intervals $I \subset \mathbb{R}$ as follows.

The algebra $\mathscr{U}_{I}(G)$ is defined to be the $C^{*}$-algebra generated by the set

$$
\left\{\tau_{s}(A) ; A \in G, s \in I\right\} .
$$

Thus $\mathfrak{I}_{I}(G)$ corresponds to the algebra generated by the orbits of the set $G$ corresponding to the interval $I$.

Next note that for each $t \in \mathbb{R}$ we may define a shift $T_{\mathfrak{t}}$ acting on $\mathfrak{A}$ by

$$
\begin{aligned}
T_{\imath}(A) & =\tau_{\imath}(A), \\
T_{\imath}^{n}(A) & =\tau_{n t}(A), A \in \mathfrak{A} .
\end{aligned}
$$

We will associate with each such shift and the set $G$ a second subalgebra $\mathfrak{A}_{1}(G)$ of $\mathfrak{A}$ as follows.

The algebra $\mathfrak{A}_{t}(G)$ is defined to be the $C^{*}$-algebra generated by the set

$$
\left\{T_{t}^{n}(A) ; A \in G, n \in \mathbb{Z}\right\} \text {. }
$$

Thus $\mathfrak{A}_{t}(G)$ is really the algebra generated by the orbits of $G$ under the shift $T_{\text {. }}$

A third useful algebra is defined in terms of the generator $\delta$ of $\mathfrak{I}$, i.e. the derivation given by

$$
\delta(A)=\lim _{t \rightarrow 0}\left(\tau_{i}(A)-A\right) / t
$$

where $D(\delta)$ is the set of $A$ such that the limit exists. If $G$ is a finite subset of $\mathfrak{U}$ and each $A \in G$ is such that $A \in D\left(\delta^{n}\right)$ then we define $\mathfrak{A}(\delta ; G)$ as the $C^{*}$-algebra generated by

$$
\left\{\delta^{n}(A) ; A \in G, n=0,1,2, \cdots\right\} .
$$

These algebras have a tendency to be equal. We next give a general result of this nature. First recall that $A \in D(\delta)$ is bounded, or geometric, with respect to $\delta$ if $A \in D\left(\delta^{n}\right)$ for all $n$ and

$$
\left\|\delta^{n}(A)\right\|<\left(r_{s}(A)\right)^{n}\|A\|
$$

for some $0<r_{\delta}(A)<+\infty$ and all $n=1,2, \cdots$.

THEOREM 4. Adopt the above assumptions and definitions.

It follows that

$$
\mathfrak{A}_{\imath}(G) \supseteq \mathfrak{A}(\delta ; G), \quad I \ni 0 .
$$

Next assume that all elements $A \in G$ are bounded with respect to $\delta$ and introduce $r_{5}(G)$ by

$$
r_{\delta}(G)=\sup _{A \in G} r_{\delta}(A)
$$


It follows for $0<|t|<1 / r_{\delta}(G)$ and $I \neq \phi$ that

$$
\mathfrak{A}_{l}(G)=\mathfrak{A}(\delta ; G)=\mathfrak{A}_{1}(G) .
$$

In particular $\mathfrak{H}_{I}$ is independent of $I$ and $\mathfrak{A}_{t}$ is independent of $t$ (in the range $\left.0<|t|<1 / r_{\gamma}(G)\right)$.

PROOF. The first statement of the theorem is straightforward to prove. As $I \ni 0$ one has

$$
\delta(A)=\lim _{t \rightarrow 0}\left(\tau_{t}(A)-A\right) / t \in \mathfrak{A}_{l}(G)
$$

and the result follows by iteration. The proof of the second statement is longer and we relegate it to the appendix.

Now we give some examples of applications of this type of criterion. For simplicity we consider a one-dimensional spin $-\frac{1}{2}$ system, i.e. the vector space $\mathscr{K}_{x}$, associated with each point $x \in Z$, is two-dimensional. The $C^{*}$-algebra $\mathfrak{N}_{\{x\}}$, of $\mathfrak{A}$, can be parametrized in terms of Pauli matrices $\sigma_{x}^{(1)}, \sigma_{x}^{(2)}, \sigma_{x}^{(3)}$, and the identity $\mathbb{1}$. The algebra $\sigma_{\mathrm{A}}$ then corresponds to the polynomial algebra generated by $\left\{\mathbb{1}, \sigma_{x} ; x \in \Lambda\right\}$.

THEOREM 5. Consider the one-dimensional generalized Heisenberg model, i.e. a spin $-\frac{1}{2}$ system with interaction

$$
\Phi(\{x, x+1\})=\sum_{i=1}^{3} J_{i} \sigma_{x}^{(1)} \sigma_{x+1}^{(1)}
$$

and $\Phi(X)=0$ if $X \neq\{x, x+1\}$ for some $x \in Z$.

Let $\mathfrak{P}^{k, x}$ denote the $C^{*}$ algebra generated by the set of elements

$$
\left\{\tau_{\imath}^{\Phi}(A) ; t \in[-\varepsilon, \varepsilon], A \in \mathfrak{A}_{\{x, x+1\}}\right\} .
$$

It follows that

either two $J_{1} \neq 0$ and then

$$
\mathfrak{P}^{\varepsilon, x}=\mathfrak{A} \quad \varepsilon>0
$$

or at most one $J_{t} \neq 0$ and then

$$
\mathfrak{A}^{\varepsilon, x} \subset \mathfrak{A}_{\{x-1, x, x+1, x+2\}} .
$$

Proof. One has that

$$
\begin{aligned}
\lim _{i \rightarrow 0}\left(\tau_{t}^{\Phi}\left(\sigma_{x}^{(1)}\right)\right. & \left.-\sigma_{x}^{(1)}\right) / t \\
& =\delta\left(\sigma_{x}^{(1)}\right) \\
& =i \sum_{X}\left[\Phi(X), \sigma_{x}^{(1)}\right] \in \mathfrak{A}^{\varepsilon, x} .
\end{aligned}
$$


By explicit calculation one finds that there is an $A \in \mathfrak{U}^{\mathrm{r}, \mathrm{x}}$ such that

$$
\delta\left(\sigma_{x}^{(1)}\right)=A+2\left(J_{3} \sigma_{x-1}^{(3)} \sigma_{x}^{(2)}-J_{2} \sigma_{x-1}^{(2)} \sigma_{x}^{(3)}\right) .
$$

Therefore

$$
4 i J_{3} \sigma_{x-1}^{(3)}=\sigma_{x}^{(1)}\left[\delta\left(\sigma_{x}^{(1)}\right)-A, \sigma_{x}^{(3)}\right] \in \mathfrak{A}^{\varepsilon . x} .
$$

The assumption that two $J_{1} \neq 0$ then implies that two of the $\sigma_{x-1}^{(i)} \in \mathfrak{A}^{\varepsilon, x}$. The third component is then obtained, up to a factor, as the product of the first two and is itself an element of $\mathfrak{Y}^{\varepsilon, x}$. Therefore $\mathfrak{P}^{\varepsilon, x} \supset \mathfrak{A}_{\{x-1, x+1\}}$. Iteration of this argument then yields the first statement of the theorem.

The second statement of the theorem follows by explicit computation of the $\tau_{t}^{\phi}$.

Remark. It is also possible to add an external field interaction $\Phi(\{x\})=$ $h \sigma_{x}^{(3)}$ or consider more general range interactions combined with larger subalgebras of observables to obtain a similar division of interactions into two classes.

The moral of this last theorem is that the Heisenberg interactions are divided into two species. Either only one $J_{1} \neq 0$ (the Ising model) and the algebra generated by the translates $\left\{\tau_{:}^{\Phi}\left(\mathfrak{H}_{\{x, x+1\}}\right) ; t \in[-\varepsilon, \varepsilon]\right\}$ is small or two $J_{1} \neq 0$ (the $X-Y$ model, Heisenberg model) and the corresponding algebra is large, $\mathfrak{I}^{\varepsilon, x}=\mathfrak{I}$. In the first case the interaction does not mix the system, i.e. cause dispersion, but in the latter case there obviously must be some form of dispersion. This form of phenomenon is similar to that encountered in relativistic field theory which gives rise to the Reeh-Schlieder theorem (see for example [5]). Thus the algebras introduced in this section have a tendency to be equal (Theorem 4) and a tendency to be either large if the interaction has a dispersive character or small if there is no such effect.

Finally we mention without explicit proof that the conditions of Theorem 4 can be completely verified for the $X-Y$ model (two $J_{1} \neq 0$ ); the shift algebra $\mathfrak{N}_{t}(G)$ with $G=\mathfrak{A}_{\{x, x+1\}}$ has the property that $\mathfrak{A},(G)=\mathfrak{N}$ for $0<|t|<t_{c}$ and $\mathfrak{R}_{t}(G) \neq \mathfrak{I}$ for $|t|>t_{c}$. The initial value $t_{c}$ is related to the group velocity $V$ of the system by $V=1 / t_{c}$.

\section{Appendix}

This appendix is devoted to the proof of the second statement of Theorem 4.

Lemma 2. Adopt all the assumptions of Theorem 4.

It follows that 


$$
\mathfrak{N}_{l}(G)=\mathfrak{A}(\delta ; G) \supseteq \mathfrak{U}_{t}(G)
$$

for all $I \subset \mathbb{R}(I \neq \phi)$ and all $t \in \mathbb{R}$.

Proof. The algebra $\mathscr{H}_{1}(G)$ will contain the elements $\delta\left(\tau_{t}(A)\right)$, $\delta^{2}\left(\tau_{t}(A)\right), \cdots$ for all $A \in G$ and $t \in I$ because these elements can be uniformly approximated by elements of $\mathfrak{R}_{I}(G)$. As $A$ is bounded, and hence $\tau_{t}(A)$ is bounded, one may construct $\tau_{s} \tau_{t}(A)$ by a uniformly convergent sum (note that $\delta$ is closed). Hence $\tau_{t}(A) \in \mathfrak{Q}_{I}(G)$ for all $t \in \mathbb{R}$ and $A \in G$. Repeating the first step of this process around the point $t=0$ we conclude that $\delta(A), \delta^{2}(A), \cdots \in \mathfrak{I}_{I}(G)$. Hence $\mathfrak{A}_{I}(G) \supseteq \mathfrak{A}(\delta ; G)$.

The reverse inclusion is also evident because $\tau_{t}(A)$ may be constructed within $\mathfrak{I}(\delta ; G)$. This also shows that $\mathfrak{A}_{t}(G) \subseteq \mathfrak{A}_{r}(G)$. To prove the reverse inclusion for small $t$ we first need the following:

Lemma 2. Let $\delta$ be the infinitesimal generator of a group $\tau$ of automorphisms of $\Re$. Let $A$ be bounded for $\delta$.

If follows that

$$
\left\|\left(\tau_{i}-1\right)^{n}(A)\right\| \leqq\left(r_{\delta}(A)|t|\right)^{n}\|A\| .
$$

Proof. Let $F_{B}$ be defined by

$$
\begin{aligned}
F_{B}(t) & =\left\|\left(\tau_{t}-1\right)(B)\right\| \\
& =\left\|\tau_{t / 2}\left(\tau_{t / 2}-1\right)(B)+\left(\tau_{t / 2}-1\right)(B)\right\| \\
& \leqq 2 F_{B}(t / 2) .
\end{aligned}
$$

Therefore with $t_{n}=t / 2^{n}$ one has

$$
F_{B}(t) \leqq|t| F_{B}\left(t_{n}\right) /\left|t_{n}\right| \text {. }
$$

Taking $B \in D(\delta)$ one may pass to the limit $n \rightarrow \infty$ and one then finds

$$
F_{B}(t) \leqq|t|\|\delta(B)\| \text {. }
$$

Thus

$$
\begin{aligned}
\left\|\left(\tau_{t}-1\right)^{n}(A)\right\| & \leqq|t|\left\|\left(\tau_{t}-1\right)^{n-1}(\delta(A))\right\| \\
& \leqq|t|^{n}\left\|\delta^{n}(A)\right\| \\
& \leqq\left(r_{\delta}(A)|t|\right)^{n}\|A\|
\end{aligned}
$$

by recursion.

Lemma 3. Adopt the assumptions of Lemma 2.

It follows that for $|t|<1 / r_{\delta}(A)$ one has 


$$
t \delta(A)=\sum_{n \geq 1} \frac{(-1)^{n-1}}{n}\left(\tau_{1}-1\right)^{n}(A) .
$$

Proof. Consider the power series given in the Lemma. Using Lemma 2 the $n$-th term of this series is bounded by $\left(r_{\delta}(A)|t|\right)^{n}\|A\| / n$ and hence the series converges uniformly for $|t|<1 / r_{\delta}(A)$. The value of the series at $t=0$ is zero and

$$
\begin{aligned}
\left.\frac{d}{d t} \sum_{n=1} \frac{(-1)^{n-1}}{n}\left(\tau_{t}-1\right)^{n}(A)\right|_{t=0} & =\left.\sum_{n \succeq 1}(-1)^{n-1}\left(\tau_{t}-1\right)^{n-1} \tau_{t}(\delta(A))\right|_{t=0} \\
& =\delta(A) .
\end{aligned}
$$

All higher derivatives vanish at $t=0$ and hence we may identify the series with $t \delta(A)$. This completes the proof of the lemma.

Let us return to the proof of the theorem. From Lemma 3 it follows that $\delta(A) \in \mathfrak{A}_{t}(G)$ whenever $|t|<1 / r_{\delta}(G)$ and $A \in G$. Further one may deduce that $\delta^{M}(A) \in \mathfrak{N}_{,}(G)$ for the same range of $t$ by application of this lemma. Hence

$$
\mathfrak{I}(\delta ; G) \subseteq \mathfrak{H}_{t}(G)
$$

for $|t|<1 / r_{s}(G)$ and this result together with Lemma 1 establishes the statement of the theorem.

\section{References}

[1] E. Lieb and D W. Robınson, Commun. Math. Phys. 28. 251 (1972).

[2] K. Jakobs, 'Ergodic Theory', Aarhus Lecture Notes (1965).

[3] D W Robinson, Commun. Math. Phys. 7. 337 (1968).

[4] D. Ruelle, Statistical Mechanics, Benjamin (New York) 1969.

[5] R. F. Streater and A. S Wightman: TCP, Spin and Statistıcs. and all that, Benjamin (New York) 1964.

Département de Physique

Université d'Aix-Marseille II, Luminy, Marseille

Centre de Physique Théorique, CNRS, Marseille

France

and

Department of Mathematical Physics

University of Adelaide

South Australia, 5001. 